\title{
Analysis on the Integration of Business Intelligence and Business Process Management
}

\author{
Weiwei Zhao \\ Party School of the Central Committee of C.P.C \\ Beijing, China 100005
}

\begin{abstract}
This paper aims to discuss and analyze the common points and their respective limitations of business intelligence and business process management, and the relationships between business intelligence and business process management. According to the relevant theoretical ideas from the literature, it illustrates the necessity of the integration of the two items with the experience of business intelligence and business process management and other fields of science and technology.
\end{abstract}

Keywords-business intelligence; business process management; decision support system; integration

\section{INTRODUCTION}

Business Intelligence (BI) is a concept proposed by the Gartner Group in 1996. It includes a series of applications, infrastructure and application tools that enable technology to make the enterprises read and analyze information data to enhance and optimize the decision and performance. Russell Ekoff argues that the business intelligence is linked to all the categories of human mental content (people's understanding on the relationships, paradigms, normative data, information, knowledge, and wisdom). So it also should examine the knowledge workers Pete Drucker often refers to. And then, it is convenient for us to have a better understanding of the business intelligence.

A master of decision support systems and business intelligence, Dan Bauer argues that business process management (BPM) refers to the use of the specific information system to improve business processes (such as planning and forecasting) to assist the managers in determining, measuring and managing corporate performance in accordance with strategic objectives. The business objectives are translated into key performance indicators (KPIs) during the management process, which are manipulated by the computer system. A computer-based dashboard is a tool of BPM or Enterprise Performance Management (CPM). Gartner argues that BPM is a way. It sees the business process as an asset that can directly improve the business performance through superior operations and business flexibility. A simple principle of the business process is that companies can achieve their goals through a series of behaviors. There are two different concepts that need to be mentioned. The first is the efficiency of the ratio between the amount of the actual result and the budget amount. And the second is the efficiency which is usually defined as the ratio between expenditure and income as shown in "Fig. 1". The two concepts are often used in the management. For the enterprises, it is difficult to improve the efficiency and interests in the same time.

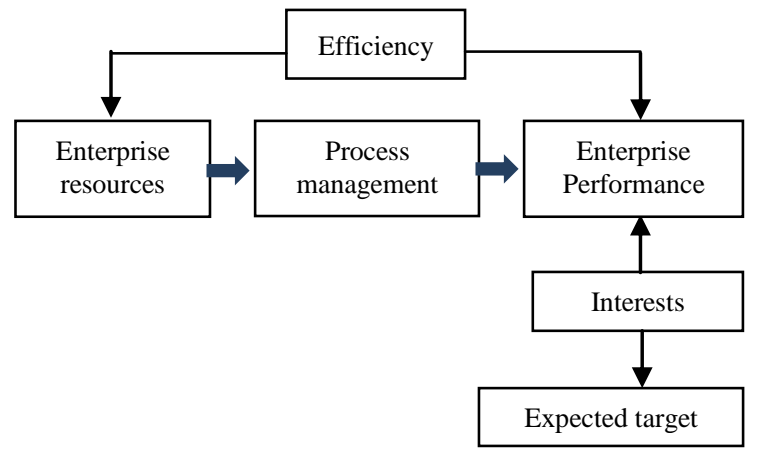

Fig. 1. Schematic diagram of efficiency and Interests.

\section{DECISION SUPPORT SYSTEMS AND BUSINESS INTELLIGENCE}

The application of Decision support system (DSS) is earlier than business intelligence. And it can be regarded as the predecessor of business intelligence. The decision support system was first introduced in 1971, proposed by Goris and Scott Morton. They argue that information system management should primarily focus on making the structured decisions. And semi-structured decision and unstructured decision are defined as decision support systems. In 1992, the Australian Computer Society ranked the decision support system in the cross-cutting areas of computer science, information systems, business and business management. Techtarget.COM defines decision support systems as a computer application. It analyzes enterprise data and makes a conclusion. And then, users can make decisions more easily. It is an information application that differentiates operating the application. And it collects data in the operations of the enterprise in daily life. It reproduces information in the form of graphics, including expert systems and artificial intelligence with the corporate executives and other knowledge workers as target groups. In 2005, Arnott and Pelvan made a critical analysis on the DSS system and proposed a simple evolutionary model as shown in "Fig. 2". 


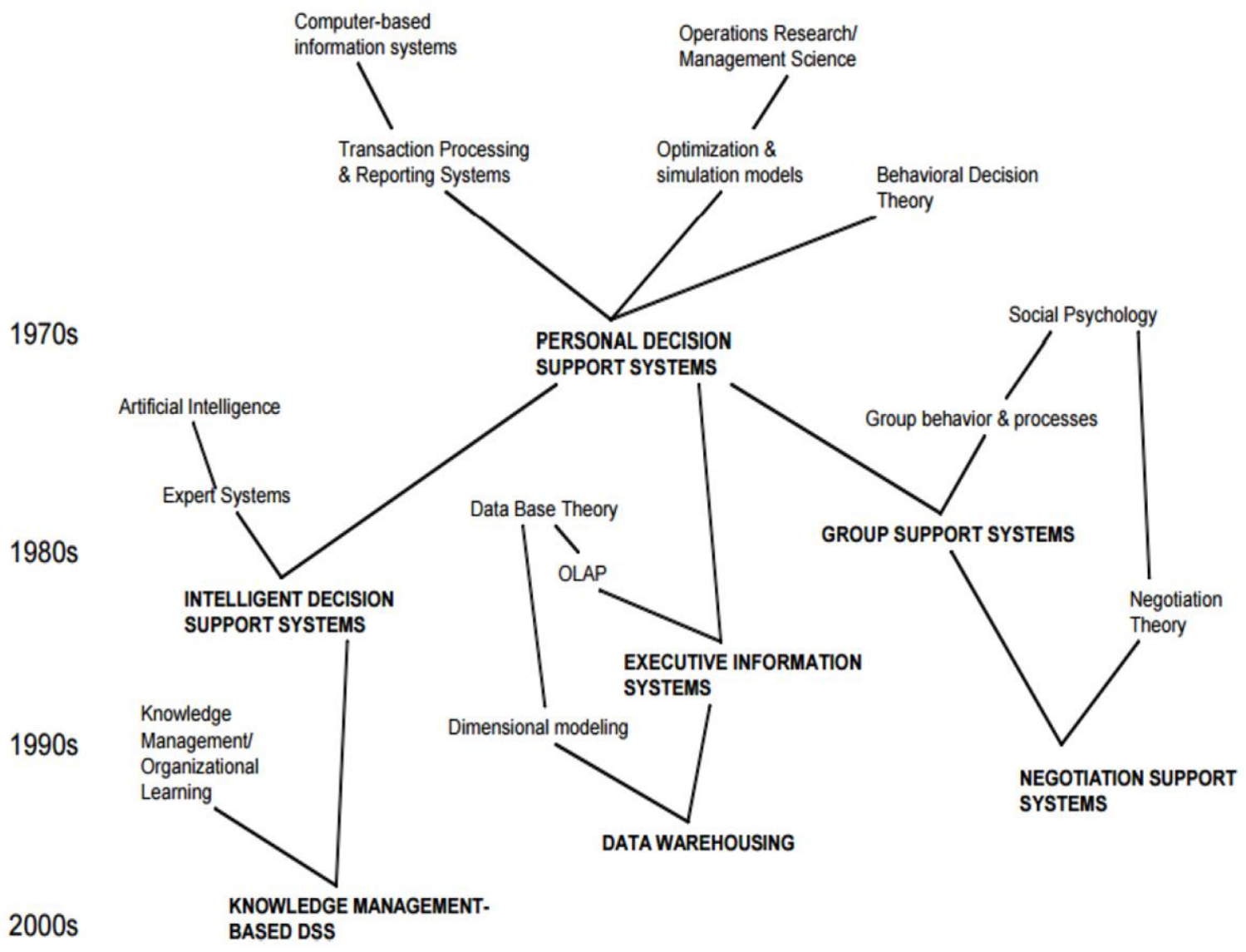

Fig. 2. DSS system domain evolution table.

Business intelligence as industry terminology was determined by Howard de Rene in 1989. In order to find a better definition of a large number of information analysis of the terms, many people used DSS system and EIS system. And they were originally used in the software industry. Dan Pauer argues that business intelligence is a series of concepts and methods for improving decision through the use of factbased support systems. Sometimes, business intelligence is interchanged with presentations, reports, inquiries, and managerial information systems. It is a data-driven decision support system. It was three years earlier than the concept of the Balanced Scorecard, which introduced the concepts such as KPI, efficiency, interests. And it has improved the ability of managers to actually implement their strategies.

Before the application of business intelligence appeared, the first generation of DSS was widely used. It consists of a series of computer tools that assist the manager in making decisions. The application of DSS system is narrower than that of business intelligence. And it was initially used by only a group of managers. Through the technological innovation, DSS system is more widely used. The number of users also increases significantly. It has further expanded the use of DSS system. And it promotes the business intelligence system to be more flexible.

Many studies and cases have proved that well-designed, appropriate, computerized decision support systems can improve decision quality, and further improve the efficiency and interests in decision process. And it makes the decision based on the facts. Decision makers pay more attention to the current information. And they ignore or forget about historical information. However, it is obvious that this unnoticed historical information hides clear trends, relationships and other patterns.

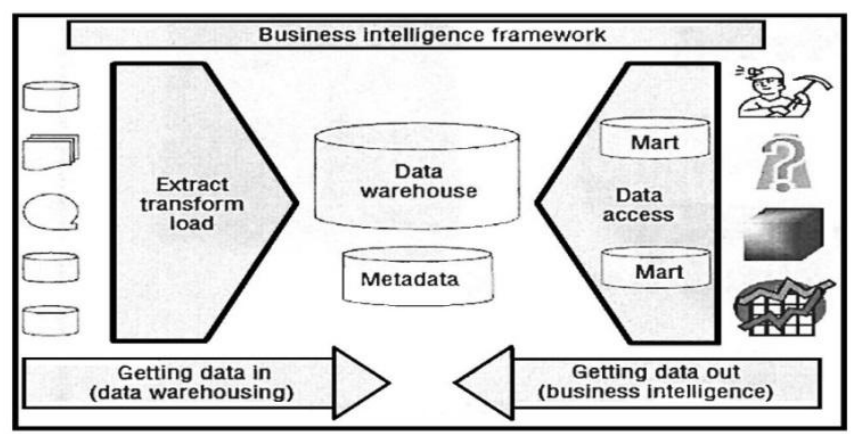

Fig. 3. BI frame structure.

Chowdhury and others believe that data integration, cleanup and standardization of these works to prepare for the $\mathrm{BI}$ is very challenging. And effective data loading is necessary as shown in the left part of "Fig. 3". In addition, when it uses the OLAP tool, the interface based on prepolymerization / precomputed data analysis shows that the reasonable speed of the view is critical. For example, the average displayed at the intersection of the geographical 
location (country, province, city, region, street) and time (year, month) by level.

In 2007, Watson and Waxham pointed out that the participation of senior management was also a very important factor for an effective implementation of the BI system. Advanced management should have BI systems. They need to provide the necessary resources and adhere to the use of information-based decision. The use and analysis of information should be a part of the corporate culture. The two scholars also believe that the enterprises need to implement the BI system, and then they also should to make training for staff. And then they can use the system. In 2008, Elbor Hill and others believe that the advantages of BI systems will take some time to fully reflect. In the early time, they are only used as a tool to making the strategic decisions. Business systems are now used in tactics and operations such as the supply chains, production and customer service. Watson and Vickham argue that BI replicates data in an independent data mart outside the enterprise during the data concise process. And it reduces the cost of IT infrastructure and eliminates data redundancy. They claim that one of the most important benefits of using BI is to help to find the right strategy when it opens up new markets or businessoriented products that are centered around the product center. Gash believes that the main influences and advantages of the BI system can be divided into several concepts. First, it can predict and find the direction of future development based on historical data of past and present performance. Second, it can analyze the results of changes and alternative programmes. Third, it can seek out specific and nonconventional solutions through temporarily obtaining the relevant data. Fourth, it deals with the problems in a strategic vision. Kurt Beck and Urik argue that the BI can be used to analyze and discover the enterprise development trends and anomalies through operational data in different business environments. And it also can run simulation programs and has an insight into business issues early. In 2010, Tuzner lists several of the common values of BI systems in his book. It can help the development of the business and organization aligned with the main goals. It can enhance the ability of analysts and managers to grasp facts and achieve to make faster decisions based on the facts to meet or exceed customer expectations on actual information. It finds potential problems by creating charts of invisible information, and it can find instantaneous solutions to new problems that can only be discovered by viewing data. It can be helpful to the cognition of competitive advantage of enterprises. And it also can be used to integrate all aspects of data to make decisions. What's more, it can collect and distribute necessary data and statistics effectively.

Watson and Wickham think that the current applications and databases of BI system are Internet systems with the real-time data warehouse technology and predictability analysis. Chowdhury and others demonstrate that the realtime performance of BI system increases customers' satisfaction. Because the companies can immediately make respond to current problems and processes. They also observed that Internet-based solutions were a big progress, and they could meet the needs of customers. Tuzner believes that predictive analysis plays an essential role in BI systems that are closely related to the business processes of the enterprise. And they can help companies to do operations on a daily basis. The results of the predictive analysis are used by enterprises to identify patterns and trends. And the results also can be used to predict business activity processes. The accuracy of the forecast results depends on the complexity of the data interdependence. To increase the number of variables is to get data as much as possible. It is helpful to increase the accuracy of the forecast results. The technology vendors of BI systems are trying to make their products as much as possible to meet these needs.

In summary, from the development of the definition of BI systems and DSS, it has been proved that each application tool of the information system has a development cycle. It is to solve some specific problems in the beginning. However, they have progressed in the target, patterns, processes, results and terminology.

\section{THE INTEGRATION OF BI AND BPM}

The famous contemporary theorist, Drucker personally felt the change of people's values. It is that people increasingly see the value of knowledge above all else. Drucker called them knowledge workers. Sixty years later, he confirmed that knowledge on the economy had become more important than land, labor, financial assets and other critical factors. And it entered into post-capital era. Before his death in 2005, he pointed out that the management of the 21 st century should improve the productivity of knowledge workers. And it was the most important contribution. In 2004, Gash argued that the business process emerged in the BPM literature was an unstructured input of a business intelligence system. And this system was related to conversations, charts, pictures, images, new projects, spreadsheets, videos and webs. In 2006, IBM explained the applications and the integration of the technologies, architectures and processes of BI systems. It is helpful to have an insight into the changes in business performance. In the use of specific technologies and other technologies, IBM mentions the key performance indicators, warnings, management dashboards, analytics applications, application integration, process modeling, monitoring and the real-time business intelligence. IBM also points out that one of the keys to the integration of BI and BPM is data integration, especially the metadata integration.

In the implementation of the synthesis report on the ways to optimize business processes to response to changes more effectively in 2008, IBM believes that the companies can quickly achieve the optimization successfully by taking full advantages of BI and BPM functions. From this point, the values that companies get when they use the two functions respectively are less than the values with using both functions at the same time. In addition, the organizations can create more efficient and profitable business processes with the use of information and the development of the process. According to the above views, IBM sees SOA (Serviceoriented architecture - the current form of subtle service architecture) as the foundation for the integration of BI and BPM. IBM insists that SOA expands the functionality of 
BPM. And then, it enables users to adapt and optimize business processes based on changes in situation initiatively. With flexibility, scalability and open standards, SOA can build, deploy and consolidate services to take advantage of existing investments to meet the business process development of enterprises. A continuous enterprise performance development model can be expressed in SOA lifecycle activities "Fig. 4".

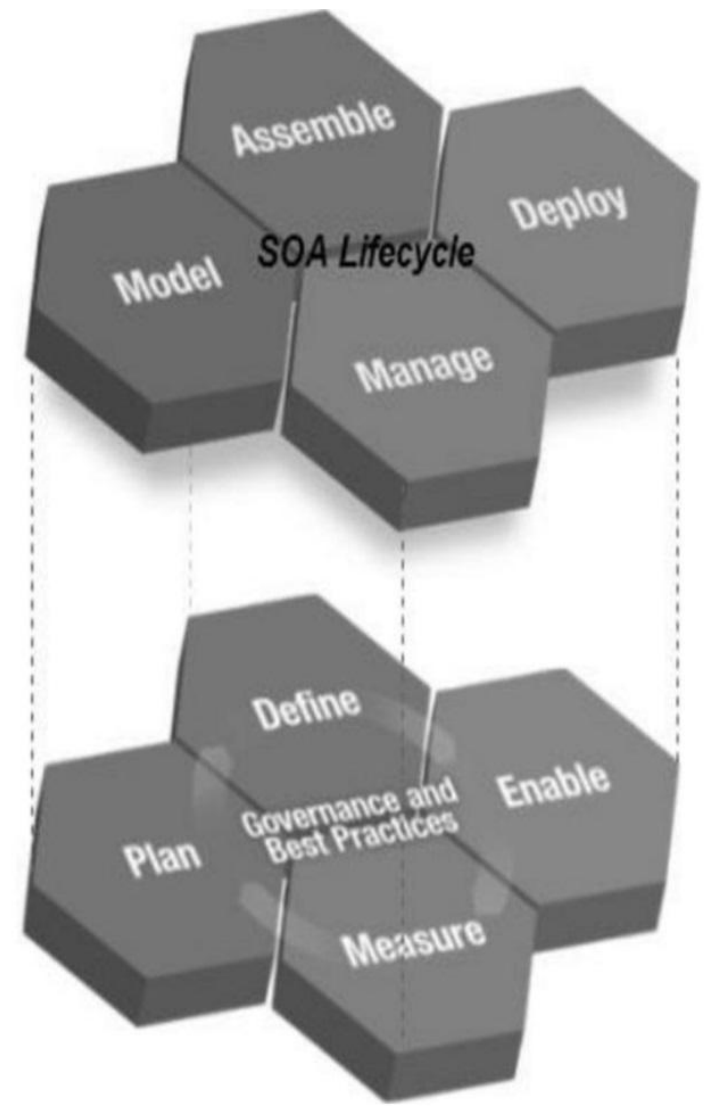

Fig. 4. A continuous enterprise performance development model.

In 2012, McKinsey pointed out that to improve the efficiency and interest in business processes is the primary goal for IT and non-IT business managers. It means that the BPM system should focus on business process modeling and automation. And then, it enables business processes to be implemented quickly and lucratively with the BI system. Moreover, the more automated the business processes are, the more interested the enterprise is in managing process execution. This is an important function of the BI system. And it can connect a variety of data resources to make the reporting mechanism more effective. In the same year, Carey Vince and kitson (Capgemini) also argued that the use of the BI analysis mechanism in business process development could make decisions quickly. And then, it can get the better results. They also recognized that the development of rules was a complex business process in the enterprise, which needs to balance the priorities of the different sectors of the organization. It is a job that requires high priority, but it is often underestimated. At Capgemini, employees were incorporated into the BPM system based on BI system, which uses a so-called process manager cockpit model to make employees similar to pilots. In 2015, they declared that they were the leaders in Intelligent Business Process Management (IBPM) on their official website. They believed that this advanced operational decision management capabilities, predictive analysis and network information integration promoted them to become the preferred partners for the digital business strategists of Accenture, Capgemini, BCG, McKinsey and other companies.

\section{REFERENCES}

[1] Zhou Ruyi. Literature review on real - time business intelligence [J]. Consumer Guide, 2016 (4): 229.

[2] Chen Lei, Dong Bidan, Zhang Feng. Operational Business Intelligence [J]. Computer Engineering and Design, 2010.31 (7).

[3] Wang Yunchao. Analysis on the development of business intelligence (BI) system [J]. Journal of Shanxi Coal Management Cadre College 2013 (10).

[4] Thomas D. Clark Jr.,Mary C. Jones, Curtis P. Armstrong. The Dynamic Structure of Management Support Systems: Theory Development, Research Focus, and Direction[M].MIS quarterly 2007, $3(3)$. 